\title{
Estrategia de Formación y Desarrollo de la Competencia Docente llamada Gestionar la Orientación Educativa Familiar, en la Educación Básica Superior
}

\author{
Leonor A. Rodríguez, Martha C. Escobar, Vicenta I. Aveiga y Ulbio C. Durán \\ Universidad Técnica de Manabí, Av. Urbina y Che Guevara, Portoviejo, Ecuador \\ (e-mail: alexanroa32@hotmail.com;marthitae65@gmail.com;vicentaaveiga@yahoo.es; uduranpico@gmail.com)
}

Recibido Feb. 10, 2019; Aceptado Abr. 5, 2019; Versión final Jun. 11, 2019, Publicado Dic. 2019

\begin{abstract}
Resumen
Se presentan los resultados de una investigación con su respectivo diagnóstico y modelado de una propuesta que propende al fortalecimiento de los vínculos familia-institución docente en la educación básica superior en Ecuador. El artículo responde a una necesidad social, propuesta por el Ministerio de Educación del Ecuador. La propuesta pretende lograr un proceso de formación continua de los docentes para que formen y desarrollen la competencia docente gestionar la orientación educativa familiar. Como resultado, se presenta una estrategia que posibilita la preparación de los docentes a través de la formación continua en la plataforma RedEducaFamilia. Su aplicación permitió constatar su efectividad en la práctica formativa mediante la implementación de diversos métodos y técnicas de la investigación. La estrategia permite también crear espacios para la reflexión, favorecer la comunicación, trabajo colaborativo entre los docentes y un cambio de actitud sobre su gestión para la orientación educativa familiar.
\end{abstract}

\section{Formative Strategy and Development of the Teaching Competence named Managing the Family Educational Orientation, in Junior High School Education}

\begin{abstract}
The results of an investigation with its own diagnostic survey and modeling of a proposal that seeks to strengthening the family-institutional links in the high school education in Ecuador, are presented. The article responds to a social need, proposed by the Ministry of Education in Ecuador. The proposal seeks to achieve a process of continuous education of teachers so that they can develop the educational competence called managing family educational orientation. As a result, a strategy that helps teachers to prepare themselves through continuous education using the platform RedEducaFamilia, was proposed. Its application allowed demonstrating its effectivity of the formative practice through the implementation of different methods and research techniques. The strategy also allows creating spaces for reflection, favoring communication, cooperative work between teachers and a change of attitude on their management for the educational family orientation.
\end{abstract}

Keywords: competences; continuous education; educational management; formative strategy; educational orientation 


\section{INTRODUCCIÓN}

La orientación educativa, concepto polisemántico, considerada como un proceso continuo (Molina, 2004), se origina en el seno de la familia, porque "... las buenas prácticas han de tener su inicio allí donde el futuro ciudadano nace y recibe los primeros cuidados, atenciones, mensajes educativos y procesos de interacción y comunicación compartida" Echeverría y Martínez (2015). Así lo reconocen autores como Christenson y Sheridan (2001); Rush y Shelden (2011); Knoche et al. (2013); y al mismo tiempo enumeran las dificultades que tiene la familia para la educación de sus hijos, especialmente los jóvenes del nivel de Educación Básica Superior, que a veces manifiestan conflictos y crisis propias de la edad a quienes los padres no prestan atención, porque no saben, no pueden, o no desean hacerlo, como sucede en determinados contextos de Ecuador; en cuanto a la orientación familiar las evidencias y soluciones desde la orientación a la familia son poco factibles en la prevención de alteraciones en el comportamiento en edades vulnerables (Lucas, 2017).

En este nivel educativo, como en otros, una de las misiones del personal docente, debe consistir en ayudar a los padres de familia a ser conscientes de la trascendencia de su tarea educativa, impulsando, gestionando y orientando la creación y desarrollo de diversas vías que incluyan su participación activa, continuada y responsable "... sin quedarnos en la mera adquisición de unos conocimientos teóricos" (Ruiz et al., 2012). Los padres no deben perder de vista, en ningún momento, que en realidad los docentes ejercen un papel complementario y de colaboración pedagógica, que requiere de un proceso de formación y desarrollo de un grupo de competencias docentes que coadyuven, al decir de Perrenoud (2012), al logro del éxito en lo que respecta a la orientación familiar y su gestión; y expresa: “... sería absurdo hacer de las relaciones entre las familias y la escuela una simple cuestión de competencias. Sin embargo, por ambas partes, un aumento de competencias podría ayudar a establecer o mantener el diálogo". La literatura consultada evidencia que las experiencias a nivel internacional relacionadas con la orientación educativa familiar no son homogéneas; en algunos contextos es función de los orientadores profesionales, grupo de orientación o tutores; en otros, de la dirección de las instituciones escolares con el asesoramiento de los orientadores o grupos de orientación y con prevalencia de un enfoque terapéutico o remedial, tal como sucede en Ecuador, específicamente en la Unidad Educativa ITSUP (nombre propio), institución con 22 años de experiencia en la formación de niños y jóvenes en educación inicial, básica, bachillerato y superior, que cuenta con una planta docente completa para cada nivel y Departamentos de Apoyo en las diversas áreas, donde a partir del diagnóstico de esta investigación se determinó que las insuficiencias en el desempeño de los docentes limitan la orientación educativa familiar en la Educación Básica Superior.

En ese sentido, los autores de esta investigación coinciden con los criterios de Berzosa et al. (2009) referido a la importancia de la participación del docente y el colectivo profesoral en la orientación educativa a la familia; y apunta: "las complicaciones de una adecuada relación padres-docentes son en buena medida las responsables de que aún no se haya podido avanzar lo suficiente en la orientación educativa familiar; es el terreno en el que falta un plan por perfeccionar (...) lo que precisa la necesidad de la formación de competencias en los docentes." Para el logro de estos y muchos otros objetivos de preparación del personal docente, es recurrente la implementación de vías de superación, entre las que se encuentra la formación continua, concepto que además ha derivado en tantos otros términos similares, pero cuyo fin radica en la formación o superación a lo largo de la vida.

A pesar de la diversidad de investigaciones efectuadas en el campo de la formación continua de los docentes (Richardson 2000; Walker et al., 2004; Jacob y Lergren, 2004; Duţă y Foloștină, 2014), se constata que aún no son suficientes los estudios realizados en lo que respecta a la preparación de los maestros para la orientación educativa familiar, que tenga en cuenta un escenario social más amplio que el del aula o la escuela, en el que mediante la realización de proyectos educativos o programas de intervención se trabaje de manera integrada con la familia y la comunidad. En otro orden, en la revisión de la literatura realizada sobre las competencias de los docentes de los niveles básico y bachillerato, se destacan los trabajos realizados por Hayes (1997); Akos (2010); Singer y Stoicescu (2011); Ezquerra et al. (2013); De-Juanas y San Martín (2014); Mirzagitova y Akhmetov (2015); quienes se dedican a la delimitación de las competencias que el profesor debe poseer relacionadas con la orientación educativa familiar; pero no se encontraron evidencias de la caracterización específica de la competencia docente gestionar la orientación educativa familiar, por lo que no fue posible precisar las particularidades para su formación y desarrollo en un inicio; aspecto que se convierte en el objetivo de este trabajo: modelación de la Estrategia docente Gestionar la Orientación Educativa Familiar a través de la Formación Continua que propenda al fortalecimiento de los vínculos familiainstitución docente en el Nivel Básica Superior en Ecuador.

\section{Formación Continua}

El contexto mundial impone la formación de profesionales que posean competencias para enfrentar las múltiples e inevitables situaciones que se dan en la sociedad actual. Por ello debe ser capaz de encontrar soluciones innovadoras, ser un autogestor de su profesión, aplicar las tecnologías con un carácter sostenible, 
desempeñarse con ética profesional, bajo la premisa de que, si es un profesional, entonces responderá con pertinencia y oportunidad a las demandas sociales y favorecerá el acceso a niveles de vida más satisfactorios para sí y para los demás.

En sentido general, la formación supone un proceso de conciencia personal, de cuestionamiento de los aprendizajes obtenidos, de indagación de respuestas teóricas y prácticas y, sobre todo, de interiorización de nuevas soluciones a problemas personales y profesionales. Mediante la formación continua (formación o educación permanente, para la vida, educación continua, aprendizaje permanente, etc.) el docente puede adaptarse a las demandas de la denominada sociedad del conocimiento, tal como lo señalan López-Barajas y Sarrate (2002); por su naturaleza cambiante, compleja y exigente, ese tipo de formación se ha convertido en una prioridad de crecimiento profesional.

La formación continua favorece en el docente el desarrollo de competencias, dominio de estrategias pedagógicas y disciplinares, reflexión y revisión responsable y comprometida sobre la práctica profesional, a través de la investigación, innovación y evaluación permanente; y, contribuye a su cualificación ulterior, promoción social, personal y empleabilidad. Igualmente se asume el criterio de Tejada (2009) que la considera como vía para el logro de la competencia docente gestionar la orientación educativa familiar, como proceso sistematizado para consolidar, reconstruir, crear saberes y desarrollar la práctica profesional que dinamice el proceso formativo escolar.

Se asumen como rasgos de la formación continua: el carácter procesual y permanente; la renovación del docente desde la perspectiva de su formación; el eje estructurador es la propia práctica docente; valoración reflexiva y crítica de las propias prácticas; corrección, comprobación y transformación de la práctica y su finalidad es el aumento de la calidad y por ende del mejoramiento del sistema educativo.

En relación a las modalidades de formación continua, Rubia y Guitert (2014), es objetivo que deban materializarse teniendo en cuenta: el lugar (local, regional, internacional); el contenido (charlas, tutorías, entornos virtuales, jornadas, cursos cortos, habilitaciones...); modalidad de estudio (on-line, presencial, personal, en grupo...). De suma importancia es el contexto (Nieva y Martínez, 2016), que ofrece multitud de posibilidades de aprendizaje porque impulsa relaciones y vínculos entre las distintas profesiones del ámbito social y es un elemento esencial cuando se trata de aspectos relacionados con la familia. En este sentido, la familia debe ser el nexo entre la enseñanza formal y la no formal, por cuanto la implicación y compromiso de todos los agentes educativos y comunitarios serán necesarios para conseguir el progreso satisfactorio del alumnado.

Otro aspecto a tener en cuenta es la incorporación de las Tic en el proceso de formación continua que debe contemplar una argumentación pedagógica sólida del por qué, para qué, cómo y dónde emplearlas. Así, bajo los lineamientos básicos del enfoque histórico-cultural de Vigotsky (1995) y sus seguidores, es posible afirmar que las Tic son un instrumento sociocultural que, dependiendo de su uso e integración educativa, influyen en los cambios cualitativos del proceso formativo.

\section{Formación y desarrollo de competencias docentes}

El concepto competencia ha tenido su evolución histórica y ha sido definido desde diversas ciencias y perspectivas, entre las que se encuentran la Psicología, la Lingüística, la Sociología y por supuesto la Pedagogía, presente en la educación del siglo XXI como concepto que señala el camino de desarrollo en la aspirada sociedad del conocimiento. Así, en la literatura consultada existen autores y organizaciones aprecian indistintamente a las competencias como capacidades, atributos, saberes, habilidades, formaciones psicológicas, conjunto de comportamientos, características esenciales, características subyacentes, capacidad y disposición humana para la actuación, repertorios de comportamientos, procesos complejos, procesos integrales, entre otros. Se coincide con el criterio de Tobón (2014) acerca de la necesidad de ofrecer un tratamiento interdisciplinario al proceso de formación y desarrollo de las competencias; no obstante, se asume como competencia, la definición aportada por este autor (p.114) al considerarlas como "Actuaciones integrales para identificar, interpretar, argumentar y resolver problemas del contexto, desarrollando y aplicando de manera articulada diferentes saberes (saber ser, saber convivir, saber hacer y saber conocer), con idoneidad, mejoramiento continuo y ética". En ello radica la socioformación, para que la persona pueda desarrollarse de manera integral, con un proyecto ético de vida sólido, en el marco de las relaciones colaborativas con otras personas, afrontando los retos futuros y actuales del contexto y contribuyendo al tejido social, desarrollo organizacional, economía, cultura y al equilibrio ecológico ambiental.

En relación a las competencias docentes, han sido también diversos los autores que las han abordado, sobre todo desde la perspectiva de su definición e identificación. Para Montes de Oca y Machado (2014) las competencias docentes son "... las configuraciones de la personalidad del profesional de la educación que lo hacen idóneo para el desarrollo de la actividad pedagógica. Constituyen el contenido principal de su modo de 
actuación y se manifiestan en el desempeño de sus funciones. Son constructos que permiten estudiar, proyectar, revelar, evaluar de manera integrada un conjunto de conocimientos, habilidades y hábitos, capacidades pedagógicas, habilidades profesionales, intereses, motivaciones, valores, normas, estilos, orientaciones y cualidades de la personalidad del docente en relación con el desarrollo de funciones específicas. El contenido de las competencias incluye componentes conceptuales, procedimentales, motivacionales y actitudinales, que en una interacción dinámica la configuran" (p.153). Estos autores ubican a las competencias docentes como contenido principal y que se manifiestan en el modo de actuación de este profesional. Por otra parte, García (2015), señala que las competencias docentes permiten solucionar los problemas inherentes al proceso pedagógico en general y al proceso de enseñanza-aprendizaje en particular en el contexto de la comunidad educativa escolar y en correspondencia con el modelo del profesional de la educación, con el propósito de promover el desarrollo integral de la personalidad de los estudiantes.

Este criterio no tiene en cuenta las tendencias vigentes de la formación escolarizada, relacionadas con la actuación del docente tanto con la familia, como con la comunidad en función de la formación de los niños y adolescentes. De ahí que se considere más apropiado el término contexto de la comunidad educativa, atendiendo a que, tanto la escuela, la familia y la comunidad forman parte del mismo. También se coincide, que el propósito de una actuación competente del docente debe estar enfocado a la promoción del desarrollo integral de los estudiantes, concepción que se asume a partir de la socioformación, y como el centro de los problemas a resolver por los docentes. Por dicha razón, atendiendo a las limitaciones de la definición de competencias docentes y desde la perspectiva de la socioformación, a partir del análisis epistemológico realizado, los autores de este artículo conciben las competencias docentes, a los efectos de rigor, como: Actuaciones integrales de los docentes para identificar, interpretar, argumentar y resolver problemas relacionados con la formación integral de los estudiantes en el contexto de la comunidad educativa (escolar, familiar, comunitaria), con idoneidad y compromiso ético, desarrollando y movilizando el saber ser, el saber hacer $y$ el saber conocer.

A partir del análisis desarrollado hasta el momento sobre las competencias y las competencias docentes se ha llegado a las siguientes consideraciones: a) constituyen, desde la perspectiva de la socioformación, actuaciones integrales de los docentes; b) están encaminadas a identificar, interpretar, argumentar y resolver problemas relacionados con la formación integral, en el contexto de la comunidad educativa; c) el desempeño debe estar caracterizado por: la idoneidad, la metacognición, el compromiso ético y el desarrollo y movilización del saber ser, el saber hacer y el saber conocer; d) el contenido de las competencias incluye componentes conceptuales, procedimentales, motivacionales y actitudinales, que en una interacción dinámica la configuran.

\section{La formación y desarrollo de la competencia docente gestionar la orientación educativa familiar}

Atendiendo la necesidad de proveer la estructura de la competencia docente gestionar la orientación educativa familiar, desde la perspectiva científica relacionadas con la orientación educativa familiar en el contexto escolar y su limitado desarrollo, García et al., (2010); a efectos de este artículo, los autores han considerado oportuno asumir la corriente integrativa de la orientación educativa (Rodríguez, 2015), la que se hace extensiva en principio a la orientación educativa familiar con sus correspondientes adecuaciones, debido a que esta se concibe en unidad con el accionar del orientador profesional, los docentes y la familia, encaminada al logro de la función orientadora de la familia para alcanzar la formación integral de los estudiantes. Desde esa perspectiva, se asume la definición aportada por Molina (2004) y se reconoce su carácter integrador al apreciarla como el proceso "... interdisciplinario y transdisciplinario sustentado en los principios de intervención preventiva, desarrollo y atención a la diversidad del alumno, cuyos agentes educativos (orientadores, padres, docentes y tutores, familia y comunidad) asumen la función de facilitar y promover su desarrollo integral para que se constituyan en seres transformadores de sí mismos y de su entorno" (p. 7).

A los efectos de este estudio, se parte de la idea que la gestión docente de la orientación educativa familiar rebasa los marcos de la función tutorial y debe ser ocupación tanto de los tutores, como del resto de los profesores del grupo estudiantil y demás agentes educativos (directivos, orientadores y otros) de manera colaborativa y mancomunada, a través de intervenciones, con la finalidad de lograr que la orientación se acerque, por una parte, a las necesidades sociales e institucionales referidas a las exigencias del proceso formativo escolar; y por otra, a las reales necesidades y características del contexto familiar y personal de cada uno de los estudiantes. En este contexto, los autores son del criterio que existe la necesidad en el Nivel Básico Superior, atendiendo a sus características, en cada grupo estudiantil se desarrollen intervenciones, donde todos los profesores, como gestores: distribuyan tareas, asignen responsabilidades a otros, gesten cronogramas de preparación, identifiquen conflictos, analicen los factores clave que determinan la acción estratégica sobre la base del trabajo colaborativo y cooperativo de los actores, entre otras acciones que se puedan desarrollar a nivel institucional en función de la orientación educativa familiar. 
Es evidente que hoy la gestión de la orientación educativa familiar adquiere nuevas y complejas dimensiones, condicionadas por las transformaciones sociales que operan, las cuales tienen una incidencia decisiva en el proceso formativo que se lleva en los centros escolares. Respecto al desempeño de los docentes, se citan los que fueron develados y argumentados en una investigación previa realizada por los autores: 1) caracterizar el entorno familiar-escolar identificando los conflictos de la cultura existente en la familia y el proyecto que se gestiona; 2) autovalorar responsablemente el desempeño propio y de la familia para el logro de la gestión educativa, debido a que le aporta al docente información valiosa al proceso de orientación familiar y a su organización. La autovaloración responsable facilita la autogestión formativa del docente; así, él precisa de la colaboración formativa del colectivo profesoral para el logro de la creación y construcción de saberes compartidos grupales que conlleven al desempeño de los docentes y el equipo de trabajo como gestores de la orientación educativa familiar; 3)utilizar dinámicas que faciliten la comunicación asertiva con los diversos agentes que se involucran en ese proceso, son procedimientos o medios sistematizados para organizar y desarrollar la actividad de grupo mediante la comunicación. Tienen el poder de activar los impulsos y las motivaciones individuales y de estimular tanto la dinámica interna como la externa, de manera que las fuerzas puedan estar mejor integradas y dirigidas hacia las metas del grupo; 4) elaborar proyectos formales e informales de orientación educativa familiar, donde se involucren los diversos actores, gestores institucionales, comunitarios y profesionales. Así, esto les permite contribuir, de manera integral, a la solución de los principales problemas y demandas derivadas de la caracterización de la familia y su entorno; 5) liderar con responsabilidad para definir, mediante la gestión de proyectos, el modelo de familia que se espera, los medios y determinación de agentes y profesionales para hacer posible su desarrollo y la evaluación del proceso a seguir, entre otros aspectos.

Una concepción sociocultural del liderazgo no puede estudiarse sin tener en cuenta los contextos relacionales socio-históricos y culturales, que orientan las conductas, así como las acciones individuales y colectivas en la convivencia escolar a partir del enfoque histórico-cultural de Vigotsky. Al ser el docente gestor un líder en este proceso, de ninguna forma anula o limita la independencia, el activismo y la creatividad de los estudiantes, la familia, los actores o los profesionales participantes; por el contrario, los estimula.

Algunas de las características de este importante desempeño de la competencia objeto de estudio son: a) Uso de la comunicación asertiva, para expresar claramente sus ideas e instrucciones y lograr que los participantes en el proceso de orientación lo escuchen y entiendan; también, saber escuchar y considerar al grupo que se dirige. b) Inteligencia emocional, definido por Salovey y Mayer (1990) como la "...habilidad para manejar los sentimientos y emociones propios y de los demás, discriminar entre ellos y utilizar esta información para guiar el pensamiento y la acción. Los sentimientos mueven a la gente, sin inteligencia emocional no se puede ser líder" (p. 189). c) Establece metas y objetivos debido a que, para un grupo, es preciso saber a dónde llevarlo. Sin una meta clara, ningún esfuerzo es bastante. Las metas deben ser congruentes con las potencialidades y capacidades del grupo; por ello, de nada sirve establecer objetivos que no se pueden cumplir. d) Planeación, para establecer un proyecto cuando se encuentra definida la meta, se deben definir las acciones, tiempo, responsables y recursos necesarios. e) Autoconocimiento de fortalezas y debilidades para hacer uso de ellas o subsanarlas. Evalúa objetivamente los procesos e impactos logrados. En ese sentido, esta evaluación debe ofrecer evidencias sólidas y creíbles del desempeño del docente gestor $y$, lo que es fundamental, puede determinar que el proceso de orientación educativa a la familia ha logrado los resultados deseados. Investiga los problemas relacionados con la problemática por cuanto la familia es un escenario de análisis de suma importancia, y solo, mediante la utilización del método científico es posible comprenderla para lograr transformarla.

\section{La competencia docente gestionar la orientación educativa familiar}

Como vía para el logro del propósito descrito, se optó por una Estrategia que consta de un objetivo general, premisas, requisitos para su aplicación y fases con sus respectivas acciones. El objetivo es favorecer la formación y desarrollo de la competencia docente gestionar la orientación educativa familiar en los docentes del Nivel Básico Superior. Las premisas: a) voluntad política y proyección institucional hacia el desarrollo profesional docente; b) aseguramiento logístico y la existencia de una adecuada infraestructura tecnológica para la informatización del proceso, el uso de los recursos de la web y el diseño de espacios virtuales para el intercambio académico; c) preparación de los docentes que dirigirán la formación; d) crear las condiciones para que los docentes dispongan de tiempo para desarrollar las diversas actividades formativas. Los requisitos: a) contar con el cuerpo de docentes dispuestos a participar en la formación; b) sistematicidad y suficiencia de las actividades formativas. Es importante destacar que en caso de que algunos de los requisitos no estén dados, se recomienda implementar un momento de sensibilización y preparación de los miembros del colectivo profesoral y del facilitador. En la Estrategia se definen como actores al facilitador del proceso, el docente y el colectivo profesoral, así como otros especialistas en caso necesario; el primero como guía del proceso cuya presencia irá disminuyendo en la medida que el grupo profesoral vaya mostrando avances en la apropiación de los saberes relacionados con la gestión de la orientación educativa familiar y el resto como 
protagonistas de su propio proceso formativo. La Estrategia consta de cuatro fases: diagnóstico, proyección, ejecución y evaluación, que se interrelacionan entre sí. Estas se resumen en la Tabla 1 y se detalla en lo que sigue.

Tabla 1: La Estrategia para la formación y desarrollo de la competencia docente gestionar la orientación educativa familiar

\begin{tabular}{|l|l|}
\hline Fase de diagnóstico. & Diagnóstico de las necesidades formativas de los docentes y el colectivo profesoral. \\
\hline Fase de proyección. & $\begin{array}{l}\text { Esta fase se realiza a partir de la determinación de necesidades formativas de los docentes } \\
\text { y el colectivo profesoral. }\end{array}$ \\
\hline Fase de ejecución: & $\begin{array}{l}\text { Se enmarca en el proceso de formación continua de los docentes del Nivel Básica } \\
\text { Superior, cuyo objetivo consiste en ejecutar las acciones formativas de la etapa de } \\
\text { proyección. }\end{array}$ \\
\hline Fase de evaluación: & $\begin{array}{l}\text { Se concibe como un proceso continuo, a través de la observación del desempeño de los } \\
\text { docentes y el colectivo profesoral durante la ejecución del proceso educativo. }\end{array}$ \\
\hline
\end{tabular}

Fase de diagnóstico. El diagnóstico de las necesidades formativas de los docentes y el colectivo profesoral es la base en la que se sustenta la proyección del proceso de formación y desarrollo de la competencia. Su objetivo es determinar las necesidades formativas del docente y del colectivo profesoral en lo relacionado con la gestión de la orientación educativa familiar. Sus acciones: a) caracterización de las políticas y normativas estatales e institucionales referidas a la formación de los alumnos y al rol que le corresponde jugar a la familia en ese proceso; b) diagnóstico de las necesidades formativas del docente y del colectivo profesoral en lo relacionado con la gestión de la orientación educativa familiar. Para la determinación de las necesidades formativas se deben utilizar diversos métodos y técnicas encaminados a la valoración de la actuación individual y colectiva de los miembros del equipo profesoral durante la ejecución de las acciones y la valoración colectiva del nivel de correspondencia que existe entre la preparación individual del docente y la del equipo profesoral; se incluye además un análisis de las exigencias formativas familiares atendiendo a los resultados de la escala auto-valorativa y valorativa, llegando a consenso de las necesidades formativas de los docentes y el colectivo profesoral.

Fase de proyección. Esta fase se realiza a partir de la determinación de necesidades formativas de los docentes y el colectivo profesoral en torno a lo relacionado con la gestión para la orientación educativa familiar. Sobre esta base se determinan los objetivos formativos generales y se proyectan las acciones de la misma. Su objetivo es diseñar las acciones sobre la base de los resultados del diagnóstico de las necesidades formativas del docente y del colectivo profesoral. Tiene además objetivos formativos generales: 1) sensibilizar a los docentes acerca de la necesidad de formar y desarrollar la competencia; 2) favorecer la conceptualización de las principales categorías relacionadas con la gestión de la orientación educativa familiar, la comprensión de sus principios sobre la base de un enfoque epistemológico coherente con los retos actuales de la Pedagogía y del contexto particular; 3) diseñar, implementar y valorar proyectos, estrategias o programas en correspondencia con las necesidades formativas del contexto familiar, y las exigencias sociales e institucionales referidas a la formación de los estudiantes para contribuir a la resolución de problemas relacionados con la familia y con la práctica educativa; 4) estimular el desarrollo profesional y personal mediante acciones auto-formativas; 5) promover un pensamiento reflexivo en el proceso de preparación a partir de la práctica educativa; 6) contribuir a la propuesta de soluciones a problemas que se identifican en el proceso de análisis del contenido en estrecho vínculo con la práctica educativa; 7) potenciar el desarrollo de sentimientos, compromiso y respeto, de manera que les permita comprender a los estudiantes, familiares y otros sujetos implicados en las situaciones conflictivas, situarse en su lugar, ayudarlos y actuar en correspondencia con sus particularidades; 8) favorecer la comunicación, colaboración y cooperación que garanticen establecer relaciones adecuadas con los estudiantes y familiares; 9) valorar, autovalorar y covalorar recursos y propuestas para el perfeccionamiento constante. Las acciones se agrupan por actividades globales:

Determinación de las modalidades formativas: en atención a los requerimientos de la formación continua de los docentes en ejercicio del Nivel Básico Superior y las necesidades que generan los nuevos retos y transformaciones educacionales, se han previsto diferentes modalidades formativas para la formación y desarrollo de la competencia docente gestionar la orientación educativa familiar.

Diseño de los cursos y programas de intervención familiar: los cursos que se diseñen deben posibilitar la formación básica y especializada de los docentes en relación a la preparación pedagógica para la gestión de la orientación educativa familiar; sus contenidos deben ser determinados a partir de los objetivos particulares resultados del diagnóstico específico, teniendo en cuenta el resultado de investigaciones nacionales e internacionales relacionadas con la temática y los principales aportes de la Pedagogía. 
Creación de la red profesional docente: la red profesional docente debe posibilitar la formación de comunidades auténticas de aprendizaje, donde todos aprendan y se eduquen en correspondencia con los objetivos planteados, mediante la autorreflexión y la interacción social; técnicamente constituyen organizaciones conformadas por estudiantes, docentes y otros miembros que pueden ser de la comunidad educativa o familiar, en las cuales se llevan a cabo actividades de identificación de necesidades formativas para la orientación familiar, de resolución de problemas familiares que pueden ser compartidos para obtener experiencias, de conceptualización y ejecución de proyectos, entre otros. En lo específico, la Red EducaFamilia, elaborada por los autores de este artículo, cuenta con un bloque llamado curso, en él los docentes deben inscribirse para participar en cada uno dependiendo de las necesidades reales de formación; estos cursos están diseñados para dos grupos: aquellos que poseen formación inicial como docentes, y los profesionales de otras áreas que ejercen la docencia sin formación docente de pregrado. Esta red cuenta con chat, foros, tareas, otros, para cuestionarios, dudas, preguntas frecuentes y sugerencias; donde el coordinador, facilitadores y el equipo docente ubican como entradas o publicaciones las dudas más frecuentes relacionadas a diferentes aspectos, sobre el funcionamiento de la plataforma o el contenido de un tema, esto permite contactarse con un tutor o experto que le pudiera ayudar a despejar dudas y retroalimentar con publicaciones, documentos, comentarios, fotos, videos, que aporten al fortalecimiento del tema.

Organización del acompañamiento por pares entre los docentes del grupo: se concibe como un proceso sistemático de apoyo y colaboración, fundamentado en la reflexión sobre la práctica, cuya acción va dirigida a complementar, ayudar y apoyar a los docentes a resolver situaciones conflictivas de su práctica relacionadas con la orientación educativa familiar. El acompañamiento pedagógico constituye otra de las alternativas para favorecer la formación de los docentes, específicamente en el caso que ocupa, se propone la organización de pares de docentes partiendo del diagnóstico, de manera tal que se complementen a partir de las debilidades y fortalezas. Para su desarrollo existen tres fases: la primera posibilita la reflexión sobre la necesidad de cambio para la mejora y promover el deseo de aprender; la segunda establece los objetivos de crecimiento personal relacionados con la aplicación de los conocimientos pedagógicos y de gestión de manera consistente para un mejor desempeño; la tercera elabora un plan de acompañamiento personalizado que posibilite la posterior reflexión sobre la práctica desarrollada. Todo el proceso debe permitir estimular el crecimiento a largo plazo y la reflexión continuada para construir sobre la experiencia y contribuir a desarrollar la competencia.

Organización del trabajo en equipo con supervisión: en etapa los docentes se organizan por grupos de interés para participar en diversas actividades, se asignan tareas y uno de ellos se prepara sobre un tema específico, como ejemplo el proyecto de gestión de la orientación educativa familiar sobre casos específicos y conversen sobre cómo llevarlo a cabo mediante el trabajo de cooperación y equipo; de la misma manera ante situaciones complejas, sobre todo cuando no hay colaboración de los padres para desarrollar y llevar a la práctica la inteligencia emocional y la tolerancia en las relaciones con los otros agentes educativos con los que debe trabajar el docente para que su proyecto se ejecute con éxito.

Planificación de los talleres de reflexión: los talleres de reflexión son actividades presenciales cuyos objetivos y contenidos deben estar en correspondencia con los objetivos formativos generales, los resultados del diagnóstico y las necesidades formativas profesorales tanto individuales como colectivas. Ejemplo de talleres: Importancia de la intervención educativa para la gestión de orientación educativa familiar. Reflexiones y creencias sobre el rol del docente en la orientación educativa familiar. Integración de la familia y escuela en función del bienestar de los estudiantes. Estudio de casos de la familia "X" y propuestas de intervención. Estos permiten, la presentación de propuestas con fundamentos teóricos que avalan la labor docente en la gestión de la orientación educativa familiar, dando lugar al intercambio de puntos de vistas, opiniones, juicios, experiencias y vivencias.

Programación y organización del sistema de actividades formativas: en la programación se concibe el proceso de formación de los docentes con sistematicidad y frecuencia de las actividades; de manera que se logre su continuidad a partir de la complejidad y sistematización. De ahí que sea necesario indagar acerca de las mejores vías para integrar armónicamente las diversas modalidades académicas en un tiempo y espacio propicio para lograr los objetivos deseados para este tipo de formación.

Determinación del sistema de evaluación del proceso formativo: la evaluación es concebida como un proceso continuo a través del desempeño de los docentes y el colectivo profesoral durante la ejecución de la labor educativa. Esto es, acercar la evaluación del proceso formativo a la evaluación profesoral instituida, incluyendo dentro de esta un acápite relacionado con la gestión de la orientación educativa familiar donde se tengan en cuenta los criterios de desempeño y evidencias fundamentados.

Fase de ejecución: se enmarca en el proceso de formación continua de los docentes del Nivel Básico Superior, cuyo objetivo consiste en ejecutar las acciones formativas de la etapa de proyección. Son acciones: 1) orientar el proceso de formación y desarrollo de la competencia gestionar la orientación educativa familiar a partir de 
su importancia e impacto social; 2) realizar diagnósticos sobre problemáticas educativas existentes en su contexto de actuación escolar, mediante la aplicación de diversos instrumentos de investigación; 3) desarrollar acciones referidas a problematizar situaciones educativas, resolver problemas educativos y argumentar soluciones; 4) planificar proyectos educativos, con tareas específicas, que puede involucrar en la institución educativa a estudiantes, otros profesores y padres de familia; 5) evaluar la efectividad de los proyectos, diseñados con el consenso de los implicados en función de lograr transformaciones en el orden tanto individual como familiar; 6) comunicar los resultados con sensatez, prudencia, sabiduría y reflexión crítica, de acuerdo a las exigencias contextuales y familiares. En este momento juegan un papel importante las interacciones que se producen entre el profesor, el colectivo profesoral y los representantes familiares, por lo que las opiniones que los últimos tengan sobre la actuación individual y colectiva profesorales deben ser utilizadas como elemento importante de control y retroalimentación de los conocimientos, habilidades y valores mostrados por los docentes y el colectivo profesoral durante la implementación.

Fase de evaluación: se concibe como un proceso continuo, a través de la observación del desempeño de los docentes y el colectivo profesoral durante la ejecución del proceso educativo en el cual se enmarca la orientación familiar, exigiéndose como evidencias: informes donde se aborden las dificultades y conflictos en diversas situaciones y las estrategias, proyectos o programas de intervención idóneos para la prevención y solución de los problemas del contexto familiar, que se manifiestan en diversas situaciones educativas o sociales identificadas. Su Objetivo es valorar la pertinencia de la Estrategia y realizar las adecuaciones necesarias para su perfeccionamiento. Las Acciones: 1) análisis sistemático sobre la apropiación por parte de los docentes de los contenidos tratados a través de las diversas actividades; 2) aplicación de instrumentos que permitan obtener criterios valorativos acerca de la calidad de los cursos, talleres u otras actividades académicas; 3) valoración en torno a la forma en la que los docentes incorporan lo aprendido en su práctica pedagógica; 4) realización de los ajustes necesarios para el perfeccionamiento de la Estrategia.

\section{METODOLOGÍA}

En el proceso de la investigación, en un primer momento (periodo lectivo 2015) se realizó el diagnóstico en la Unidad Educativa ITSUP, se aplicó la técnica de la entrevista a las autoridades, docentes y miembros del Departamento de Consejería Estudiantil; en sus respectivos lugares y horarios de trabajo; a los padres de familia, en las reuniones y asistencia al plantel; los resultados evidenciaron insuficiencias en el desempeño de los docentes respecto de la orientación educativa familiar en el Nivel Básica Superior. A partir de estos resultados con la participación de cinco especialistas o expertos de la Institución: dos psicólogos, un médico, un abogado y un promotor social, que asesoraron en temas de su competencia para fortalecer la formación de los docentes, se modela la Estrategia de Formación y Desarrollo de la competencia: Gestionar la Orientación Educativa Familiar.

Para la ejecución de la estrategia (periodo lectivo 2016), se llevó a cabo diversas acciones: búsqueda de apoyo y comprensión por parte de los directivos de la Institución. Valoración de las posibilidades tecnológicas para el uso de las Tic; en esta institución existen dos laboratorios de cómputo con 20 máquinas cada uno, con servicio de internet. Solicitud de apoyo a especialistas docentes universitarios de la provincia de Manabí y profesionales del área de estudio, que comprendieron y apreciaron la magnitud e importancia del proyecto. Elaboración del entorno virtual, plataforma Educa Familia y su validación con los profesionales y especialistas participantes. Sensibilización al cuerpo docente de la Unidad Educativa, mediante charlas relacionadas con el rol de la familia en el proceso formativo y los problemas que se presentan.

La población participante en la estrategia, estuvo conformada por la totalidad de los docentes del Nivel Básica Superior, 16 (cinco de ellos sin formación profesional docente), quienes se inscribieron en la plataforma y escogieron los módulos de Pedagogía, Orientación, entre otros, de acuerdo con las necesidades de formación de cada uno. Además, se seleccionó de los reportes emitidos por los docentes y de los archivos del Departamento de Consejería Estudiantil, casos de familias de la institución con características: disfuncionalidad, debilidad en la comunicación, hijos con dificultades de conducta y aprovechamiento académico, poco cumplimiento de responsabilidades en el rol de cada uno de los miembros de la familia, que requerían la orientación educativa familiar.

Posteriormente se ejecutó el proceso de formación continua de los docentes con una frecuencia semanal de cuatro horas, con actividades y tareas a solucionar desde otros entornos como los hogares de las familias seleccionadas. Los indicadores de desempeño a tener en cuenta para esta fase estuvieron dados en los siguientes ejes procesuales con sus respectivos criterios: 1) Conceptualización del proceso de orientación educativa familiar. 2) Diseño del proceso de orientación educativa familiar. 3) Implementación del proceso de orientación educativa familiar. 4) Valoración del proceso de orientación educativa familiar. Para evaluar los indicadores de desempeño, se utilizó una lista de cotejo, escala de apreciación, tabla de especificaciones y preguntas clave, reflejados en Rodríguez (2015), con las respectivas modificaciones relacionadas con la fundamentación teórica del objeto de atención en la familia, 
Para comprobar la efectividad de la formación y desarrollo de la competencia en los docentes, se les encomendó varias tareas relacionadas con la gestión de la orientación educativa en familias, donde se evidencie de manera integrada el saber ser, saber convivir, saber hacer y saber conocer, por ejemplo: Tarea 1 de trabajo por equipos: Caracterizar la familia de un caso problemático seleccionado. Planteamiento del problema: En estos momentos estamos tratando de lograr identificar los problemas de la familia Castro Mera (nombres ficticios), por cuanto su hijo Marlon Castro, que cursa el décimo año básico, paralelo A, manifiesta graves problemas de asistencia, disciplina y aprovechamiento escolar. Primer momento: realizar una caracterización de la familia donde se tenga en cuenta en el diseño: información; variables psicosociales: ideologías, creencias, valores relativos a la infancia y adolescencia; autonomía psíquica/física; situaciones del medio social: falta de servicios de ocio y tiempo libre, sanitarios, escolares, para niños y adolescentes; índice de delincuencia; victimización; marginación; pobreza, otros; relación y organización de convivencia; recursos económicos; trabajo/ocupación; formación/educación; vivienda; participación social; aceptación social. Aplicar fichas de rastreo, consulta de documentos de planificación elaborados, entrevistas a informantes claves grupales e individuales. Diseño de instrumentos (si el caso lo amerita). Aplicación y procesamiento de información. Segundo momento: elaborar un proyecto de intervención con la familia seleccionada a partir de los resultados de su caracterización, que considere: pronóstico de los cambios, hipótesis de trabajo, propuesta de intervención, por ejemplo: Proyecto de Intervención Familiar, Grupal y Comunitario, en tres etapas: diseño, investigación y evaluación. Diseño: detección y refuerzo de los recursos disponibles dentro de la red de apoyo social; atención directa a la población; actuaciones asistenciales y de reinserción mediante el apoyo y tratamiento psicosocial en los diferentes marcos de convivencia; canalización y derivación a otros servicios o sistemas cuando la intervención lo requiera. Investigación: estudiar la coherencia y operatividad del proyecto, elaboración de instrumentos que permitan la recogida y procesamiento de información. Evaluación: validación del proyecto de intervención.

Transcurrido seis meses de la formación continua, a partir de la observación y su materialización en la lista de cotejo, se determinó la efectividad de la estrategia de Formación y Desarrollo de la competencia: Gestionar la Orientación Educativa Familiar, que ejecutó por dos periodos lectivos consecutivos 2016-2018.

\section{ANÁLISIS Y DISCUSIÓN DE RESULTADOS}

Los resultados de la observación y materialización en la lista de cotejo, escala de apreciación, preguntas clave y tabla de especificaciones, reflejaron:

El $81.5 \%$ de los docentes participantes ejecutaron los cuatro ejes procesuales del desempeño; es decir, conceptualizan, diseñan, implementan y valoran el proceso de orientación educativa familiar para detectar e intervenir en los diversos casos que se presentan en la institución. Se demuestra la pertinencia de la formación continua y la práctica de la competencia con repercusiones positivas en el ámbito de las familias atendidas.

El $62.5 \%$ (10), en cuanto a los resultados de la escala de apreciación lograron una interacción social efectiva, consiguieron determinar soluciones de manera pacífica a la problemática de las familias; mostraron iniciativa en la toma de decisiones, anticipación y proyección de los hechos; observaron una actitud positiva y compromiso con la tarea y fueron capaces de analizar las causas de sus éxitos y fracasos de forma reflexiva. Se evidencia que los docentes están capacitados para gestionar las necesidades de atención en las familias.

En la tabla de especificaciones, el $56.25 \%$ se ubica en una escala avanzada donde se pudo comprobar que, previo a la caracterización de la familia realizan análisis teóricos de la literatura sobre el tema objeto de atención; elaboran adecuadamente los instrumentos y los validan; así como utilizan los medios y recursos adecuados para procesar la información. Aspectos esenciales para el logro de la comunicación asertiva con la familia y otros agentes educativos. Se evidencia que los docentes investigan y fundamentan científicamente los casos de estudio, que permite disminuir el margen de error en su actuación.

En lo que respecta a las respuestas ofrecidas a las preguntas clave, el $75 \%$ (12), pudo responder adecuadamente los procesos seguidos para la ejecución de las acciones asignadas, lo que denota una adecuada orientación de los procesos metacognitivos de la competencia; entre ellos, pudieron explicar pasos seguidos para la caracterización de la familia, los criterios para la selección de informantes clave, procedimientos para la aplicación de instrumentos, identificación de las dificultades en el proceso investigativo llevado a cabo y determinación de indicadores. Importante fue la objetividad en la argumentación de la correlación existente entre los resultados de la caracterización efectuada y el proyecto elaborado para la solución de la problemática. Se pudo corroborar que existieron avances en el proceso de formación y desarrollo de la competencia docente gestionar la orientación educativa familiar.

De esta forma, los hallazgos del estudio se relacionan con lo que afirman Guevara y Herrera (2012), que para ejercer la orientación educativa, el docente no requiere ser un especialista en Psicopedagogía, sino adquirir competencias en los ámbitos cognitivo, social y el de saber ser, porque la orientación no se improvisa, debe 
prepararse científicamente para ejercerla, a través de un plan de formación permanente del profesorado con etapas de formación inicial y continua que contemple talleres que simulen la actividad de orientación para practicar la técnica y resolver dudas. Asimismo, se encontró una investigación similar realizada por Fragoso y Canales (2009), acerca de la formación educativa en valores desde la educación informal de la familia, que identifica como método de intervención la investigación-acción, para promover la solución de problemas, desde la planeación hasta la evaluación del mismo que permitirá al docente auto-observarse constantemente y que sea crítico ante su propio actuar. Esta recomendación coincide con el estudio realizado por Vezub (2007) donde indica que este tipo de formación resulta ser más efectivas por el trabajo colaborativo, situacional e institucional que generan, porque es este espacio de trabajo donde surgen y se pueden resolver la mayor parte de los problemas. De este modo, se detectan situaciones reales y significativas que se convierten en objeto de reflexión y análisis, y el establecimiento de nuevos vínculos entre el docente, el conocimiento y su práctica.

Los resultados obtenidos en este estudio aportan una visión particular sobre la formación y desarrollo de la competencia docente Gestionar la Orientación Educativa Familiar, desde un proceso de sensibilización y de conceptualización hasta la resolución de problemas mediante un proceso colaborativo-valorativo de la gestión de la orientación educativa familiar. Esta visión coincide con el estudio realizado por Cánovas et al., (2014) relacionado con la Estrategia de Intervención Socioeducativa con familias que consideró que la mediación familiar como estrategia, en el abordaje de problemáticas familiares no alcanzó valores importantes en esa población objeto de estudio, y les preocupó que, siendo una estrategia con un potencial educativo importante, tuviera un menor impacto en el trabajo con familias. Consideraron necesario continuar trabajando en este sentido en la formación de los profesionales, que incluye a los profesores. De la misma manera con el objetivo de evaluar las posibilidades del Programa de Formación de Padres, PECES/STEP, desarrollada por la Universidad del País Vasco, Bartau, et al., (2001) en un contexto sociocultural para responder a las demandas de formación de padres y mejorar las relaciones padres- hijos, identificaron que esta formación debe empezar con los profesores, lo que se complementa con lo manifestado por Lucas (2017) en la Estrategia Educativa de Orientación Familiar para la atención a las carencias afectivas en el desarrollo del escolar ecuatoriano, donde para su implementación tiene en cuenta a todos los agentes que directamente se vinculan con los estudiantes aunque centre su atención en los padres de familia, pero no es privativo de ella. Por otro lado, los resultados del estudio son distintos con los hallazgos de De Bofarull (2010) que, en opinión de la autora, de la tesis doctoral: Bases para una Orientación Familiar de Fortalezas y Competencias, Universidad de Barcelona España, su propuesta no deber ser llevada a cabo por los maestros o profesores que conviven a diario con los alumnos y las familias sino por orientadores especializados externos a la escuela.

\section{CONCLUSIONES}

A partir de la reconstrucción conceptual de la competencia docente gestionar la orientación educativa familiar, se modela la estrategia como un sistema que se enmarca en el proceso de formación continua de los docentes, incluye un sistema de formación y autoformación, y tiene como funciones esenciales su transformación cualitativa, potenciación y desarrollo. Gracias a ello, mejora su profesionalidad como gestor de la orientación educativa familiar, creador de una comunidad profesional de aprendizaje, participante de entornos interactivos, redes profesionales, trabajo colaborativo, otros, que significó un salto cualitativo en el desempeño profesional, porque si bien al inicio, ningún docente reconoció que era capaz de gestionar ese proceso, se comprobó en las tareas asignadas, que su gestión respondía a la solución de los problemas de la familia seleccionada, aun con limitaciones en la caracterización efectuada y en los proyectos elaborados.

La aplicación de la estrategia, durante dos periodos lectivos, refleja organización y estructura que desde sus premisas, requisitos y acciones permite asegurar que los docentes formados continúen con actitud proactiva en la solución de diversos problemas del contexto educativo. Entre sus posibles limitaciones se identifica que el diseño no contempla la inserción de docentes recién incorporados a la institución, así como el debilitamiento del compromiso institucional y de la familia para continuar con el fortalecimiento de la relación escuela-familia.

\section{REFERENCIAS}

Akos, P., Moving through elementary, middle, and high schools: The primary to secondary shifts in the United States, Educational Transitions. Moving Stories from Around the Word, 125-142 (2010)

Bartau, I., J .Maganto., y J. Etxeberría. Los programas de formación de padres: una experiencia educativa. Revista iberoamericana de educación, 25(1), 1-17(2001)

Berzosa, M.P., V. Cagigal De Gregorio y I. Fernández-Santos Ortiz, El reto de la orientación familiar en los centros educativos. Una realidad que necesita mejorar, Revista Apuntes de Psicología, 2-3(27), 441-456 (2009) 
Cánovas P., P.M. Sahuquillo, E. Císcar y C. Martínez, Estrategias de intervención socioeducativa con familias: Análisis de la orientación familiar en los servicios especializados de atención a la familia e infancia de la comunidad valenciana, doi: 10.5944/educxx1.17.2.11491, Educación XX1, 17 (2), 265-288 (2014)

Christenson, S.L, y S.M. Sheridan, Schools and families: Creating essential connections for learning. New York: Guilford Press Estados Unidos (2001)

De Bofarull, I, Bases para una orientación familiar de fortalezas y competencias, Tesis Doctoral, Universidad de Barcelona España (2010)

Duţă, N.V. y R. Foloştină, Continuous training of university teachers - theoretical approaches and practical implications, doi: 10.1016/j.sbspro.2014.01.781, Procedia - Social and Behavioral Sciences 116, 3449-3453 (2014)

Echeverría, B., y P. Martínez, Luces entre sombras de la Orientación en acción, doi: 10.6018/reifop.18.2.219031, Revista electrónica interuniversitaria de formación del profesorado, 18(2) ,1-13 (2014)

Europan Comission Education and Training. Supporting teacher competence development for better learning outcomes. (2013)

Ezquerra, A., A. De-Juanas y C. San Martín, Teachers' Opinion about Teaching Competences and Development of Students' Key Competences in Spain, doi: https/doi.org/10.1016/j.sbspro.2014.01.372, Procedia-Social and Behavioral Sciences, 116, 1222-1226 (2014)

Fragoso F.E. y E. L. Canales. Estrategias educativas para la formación en valores desde la educación informal de la familia. Educere,13(44), 177-185 (2009)

García, A., La competencia didáctica de los maestros de informática de la educación primaria, Atenas Revista Científico Pedagógica, 31(3), 8-22 (2015)

García, M.P., M.A. Gomáriz, M.A. Hernández y J. Parra, La comunicación entre la familia y el centro educativo, desde la percepción de los padres y madres de los alumnos, Educativo Siglo XXI, 28(1), 157-188 (2010)

Guevara, G.E. y J.I. Herrera, La orientación educativa y familiar. Su implicación en la formación del profesional universitario en Cuba, Gaceta Médica Espirituana Universidad Ciencias Médicas de Sancti Spiritus, 14(2), 150-156 (2012)

Jacob, B.A. y L. Lefgren. The Impact of Teacher Training on Student Achievement: Quasi-Experimental Evidence from School Reform Efforts in Chicago, Journal of Human Resources, XXXIX(1), 50-79 (2004)

Knoche, L.L, M.E. Kuhn, y J. Eum, More Time. More showing. More helping. That's how it sticks": The Perspectives of Early Childhood Coachees, Special Education and Communication Disorders Faculty Publications, doi: 10.1097/IYC.0b013e3182a219354, (26), 349-365 (2013)

López-Barajas, E. y M. Sarrate, La educación de personas adultas: reto de nuestro tiempo, $1^{\text {a }}$ Ed., Dykinson, Madrid, España (2002)

Lucas, M.M., Estrategia educativa de orientación familiar para la atención a las carencias afectivas en el desarrollo del escolar ecuatoriano. Transformación, 13(2), 221-233 (2017)

Mirzagitova, A.L. y L.G., Akhmetov, Self-development of pedagogical competence of future teacher. Internacional Education Studies, 8(3), 114 (2015)

Molina, D.L, Concepto de orientación educativa: diversidad y aproximación, Revista lberoamericana de Educación, 35(1), 1-22 (2004)

Montes de Oca, N. y E.F. Machado, Formación y desarrollo de competencias en la educación superior cubana. Humanidades Médicas, 14(1), 145-159 (2014)

Nieva, J.A. y O. Martínez, Una nueva mirada sobra la formación docente, Universidad y Sociedad, 8(4), 14-21 (2016)

Perrenoud, P., Diez nuevas competencias para enseñar: invitación al viaje, p. 96, Editorial GRAÓ, Barcelona, España (2007)

Richardson, M.O., Peer Observation: Learning from One Another. Thought and Action, 16(1), 9-20 (2000)

Rodríguez, L.A., Modelo de formación de la competencia "orientar a la familia" en los docentes de educación básica superior, Revista Didasc@lia: Didáctica y Educación, 2(VI), 181-188 (2015)

Rubia, B. y M. Guitert, ¿La revolución de la enseñanza? El aprendizaje colaborativo en entornos virtuales (CSCL), doi: 10.3916/C42-2014-a2, Comunicar, 42(XXI), 10-14 (2014)

Ruiz, I., P. Sánchez y M.E. Jorge, Familia y educación. Guía práctica para Escuelas de Padres y Madres Eficaces, $1^{a}$ Ed., p11, Consejería de Educación, Formación y Empleo, Región de Murcia, Murcia, España (2012)

Rush, D.D, y M.LL. Shelden, The Early Childhood Coaching Handbook. Brookes Publishing Company, Baltimore, United States (2011)

Salovey, P. y J.D. Mayer, Emotional Intelligence, doi: 10.2190/DUGG-P24E-52WK-6CDG, Imagination, Cognition and Personality, 9(3), 185-211 (1990)

Singer, F.M. y D. Stoicescu, Using blended learning as a tool to strengthen teaching competences, doi:10.1016/j.procs.2011.01.043, Procedia Computer Science 3, 1527-1531 (2011) 
Tejada, J., Competencias docentes, Profesorado. Revista de Currículum y Formación de Profesorado, 2(13), 1-15 (2009)

Tobón, S.T., Formación integral y competencias: Pensamiento complejo, currículo, didáctica y evaluación, 4ª Ed., p.114, ECOE Ediciones, Bogotá, Colombia (2013)

Vezub, L.F., La formación y el desarrollo profesional docente frente a los nuevos desafíos de la escolaridad, Profesorado, Revista de currículum y formación del profesorado, 11(1), 1-23 (2007)

Vigotsky, L.S., Pensamiento y Lenguaje Teoría del desarrollo cultural de las funciones psíquicas, Ediciones Fauto, Buenos Aires (1995)

Walker, B., C.S. Holling, S.R. Carpenter y A. Kinzig, Resilience, adaptability and transformability in social-ecological systems. Ecology and Society 9(2), 5 (2004) 